\title{
Effect of Translatory Motion on Dynamics Heat and Mass Transfer of Vapor Bubble Radially Oscillating in a Liquid
}

\author{
Nail Suleiman Khabeev \\ Department of Mathematics, University of Bahrain, Sakhir, Kingdom of Bahrain \\ Email: kh.nail@gmail.com
}

How to cite this paper: Khabeev, N.S. (2019) Effect of Translatory Motion on Dynamics Heat and Mass Transfer of Vapor Bubble Radially Oscillating in a Liquid. Journal of Applied Mathematics and Physics, 7, 942-947.

https://doi.org/10.4236/jamp.2019.74063

Received: March 17, 2019

Accepted: April 25, 2019

Published: April 28, 2019

Copyright $\odot 2019$ by author(s) and Scientific Research Publishing Inc. This work is licensed under the Creative Commons Attribution International License (CC BY 4.0).

http://creativecommons.org/licenses/by/4.0/

\begin{abstract}
The phase transitions and the effect on this process of two factors: relative motion and the external influence of the carrier flow (pressure perturbation) are investigated. A mathematical model describing all the stages of the phenomenon is constructed. The model includes mass, momentum and energy balance equations (both for the vapor and for the liquid) or their first integrals.
\end{abstract}

\section{Keywords}

Vapor, Bubble, Radial, Oscillations, Mass Transfer

\section{Introduction}

The study of the dynamics and heat and mass city transfer of an individual bubble is of independent interests and, at the same time, one of the steps in the investigations of bubbly mixtures [1]. In this connection we have examined the motion and radial pulsation of a vapor bubble in an unbounded incompressible liquid flow. The vapor in the bubble condenses as a result of an increase in the pressure in the surrounding flow.

The corresponding spherically symmetric problem is studied in [2]. The survey of the literature on this topic can be found in [3]. Theoretical and experimental data on the motion of gas bubbles are presented in [4]. The convective heat and mass transfer associated with flow over a bubble without radial motion were considered in detail in [5] [6] [7]. The thermal regime of vapor bubble collapse in a subcooled liquid and the effect of the relative motion on this process were previously examined in [8], where it is also possible to find a comparison of 
the numerical solutions with the experimental data (the experimental apparatus is described in [9]).

\section{Formulation of the Problem}

We assume that the bubble always preserves a spherical shape. For estimating the validity of this assumption, it is possible to employ the Weber number test

[3]: We $<<1$. We assume also the liquid to be infinite in extent and incompressible. We considered the general case of a bubble in an axisymmetric flow whose translational velocity at infinity is not constant, because in reality the friction, apparent mass, gravitational and other forces may not be equilibrated, i.e. the motion is accelerated.

We assume that the flow is potential and that there is no boundary layer separation. In [4] [10] [11] [12] this approximation was discussed and justified for intermediate Reynolds numbers $1<<\operatorname{Re}<1000$. It should be noted that in reality the region of separation is a very narrow zone in the wake of the bubble. A feature of the formulation of the boundary-value problem of bubble motion is the absence of explicit limitations on the tangential component of the velocity vector on the interphase surface. Therefore, on the range of Reynolds numbers in question the role of the viscous boundary layer which, undoubtedly, exists in a real liquid, is of little importance. Thus, it is possible to find the velocity field potential for the axisymmetric motion of the liquid [8].

In this formulation the momentum equation for the liquid has as its first integral a Gauchy-Lagrange integral. With allowance for the balance conditions imposed on the mass and momentum at the bubble surface, this integral can be written in the form of Rayleigh-Pleset equation [1].

We assume that all the thermophysical parameters of the vapor in the bubble depend only on time. If the specific heat of the liquid and its thermal conductivity are assumed to be constant, the energy equation for the liquid can be written in terms of the temperature. The local heat fluxes vary with the angle; as a result, at the surface of the bubble the evaporation (condensation) rate has different values depending on the angle. In the framework of quasi-equilibrium phase transition scheme the phase transition rate can be determined from the condition of energy balance at the bubble surface.

The system loses equilibrium as a result of a change in the pressure in the liquid at infinity. In relation to these processes it is possible to distinguish two stages in the variation of the bubble radius [1] [9]: a dynamic and thermal stage. The present approach permits the effective study of both these stages.

It is shown that the investigated process has seven similarity criteria, each of which plays definite role. The Peclet number (together with the Jacob number) is the principal dimensionless number. The value $\mathrm{Pe}=0$ corresponds to the case in which there is no flow over the bubble. Then the system of basic equations corresponds to the formulation problem of the spherically symmetric oscillations a vapor bubble [2]. 


\section{Results of the Numerical Calculations}

The system of basic equations contains one nondimensional non-steady equation and four ordinary differential equations. For numerically solving heat transfer equation we used the method of alternating interaction proposed in [13]. This method is stable and makes it possible to solve multidimensional equations of parabolic type, The difference schemes constructed have second-order accuracy.

In order to test the correctness of the model we compared the results with the experimental data. In the experiments the following phenomenon was investigated [8] [9]: a bubble rises in water at a constant velocity, and at a certain moment of time the pressure in the liquid abruptly increases.

In Figure 1, we have plotted the bubble radius as a function of time: the points represent the experimental data, and the continuous curves the results of calculations: Curve 1 was obtained in [8], and curve 2 from the corresponding calculations carried out from the complete system of equations. Clearly, our proposed model is in good agreement with experiments. In this particular case the simpler model of [8], which does not take the bubble oscillation dynamics into account, was also found to be fairly accurate. However, it is possible to have situations in which this approach can lead to significant errors. Previously, this will be the case at large values of the disturbed to the initial pressure P (in the experiment it was equal to 1.22). We note that over a short initial interval curve 2 oscillates. The amplitude of the oscillations is small and they are rapidly damped.

In Figure 2, we have plotted the bubble radius as a function of time for the case, when initial bubble radius $\mathrm{Ro}=0.1 \mathrm{~mm}$, initial pressure was 1 bar, disturbed pressure was 2 bar. The continuous curves $1-3$ were constructed for $\mathrm{Pe}=$ 0,100 and 1000, respectively. It is clear from the figure that in the dynamic stage (oscillation of the bubble radius) the flow has the effect on the function R/Ro. Depending on the Peclet number, it may become important either in the purely thermal stage or in the intermediate stage, when the inertia of the liquid and the heat transfer are equally important. When $\mathrm{Pe}<<100$ the relative motion of the bubble finds expression in the final stage of collapse. The broken curves 4 and 5 are presented on the results of calculations carried out, in accordance with the model proposed in [8], for Peclet numbers $\mathrm{Pe}=100$ and 1000 .

The temperature distribution in the liquid near the bubble at the moment at which the bubble radius reaches a local maximum is shown in Figure 3. Here curves 1 - 3 have been constructed for the three angles: $0, \pi / 2$ and $2 \pi / 3$ (for all these cases the Peclet number was taken equal to 1000). It is easy to observe that the monotonicity of the curves may vary with the angle; accordingly, these problems cannot be solved by assuming a thin temperature boundary layer: there are angles for which at certain moments of time the temperature distribution has a maximum at a distance from the surface of the bubble begins to have a significant influence on its condensational collapse only after the oscillations of 


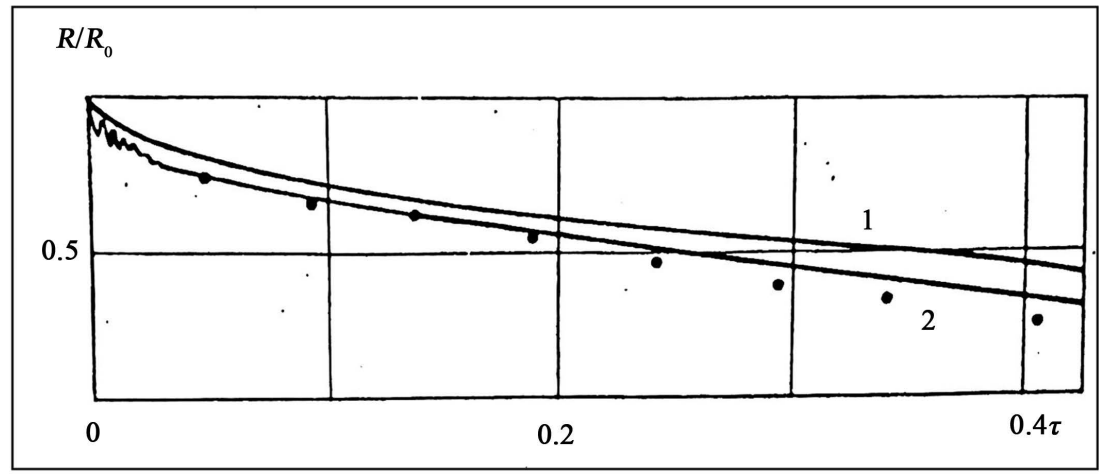

Figure 1. The collapsing bubble radius as a function of time.

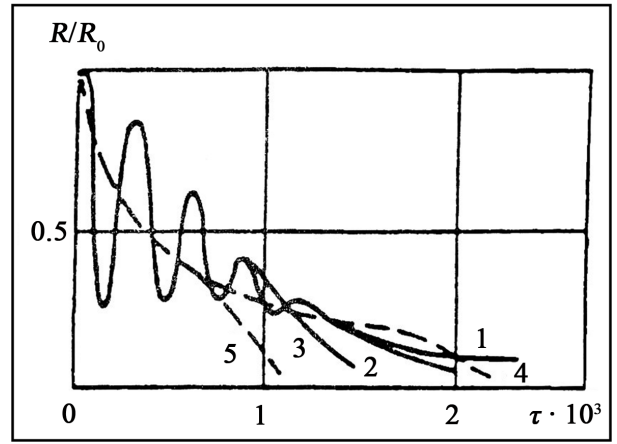

Figure 2. The collapse of the bubble for different values of Peclet number.

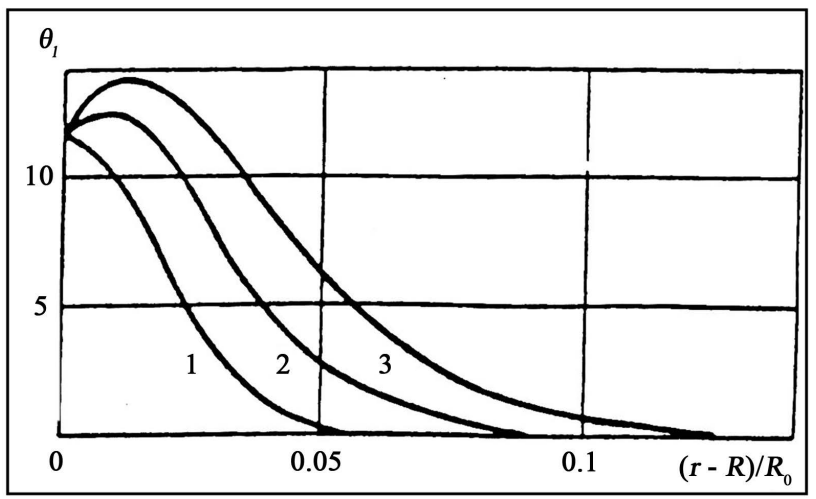

Figure 3. The temperature distribution in the liquid near the bubble surface.

the radius either cease completely or decrease considerably in amplitude, i.e. after the dynamic stage. The assumption that the pressure in the bubble adjusts itself instantaneously to the pressure in the liquid at infinity turns out to be inaccurate (see Figure 1 and Figure 2).

\section{Conclusions}

The effect of translatory motion on the dynamics of radial oscillations and heat and mass transfer of vapor bubble was investigated. Earlier this problem was studied with many simplifications particularly that translatory motion of bubble is constant. Results of numerical investigation of this problem are presented. The 
results obtained agree well with experimental data. In future studies would be interesting to study dynamics of vapor-gas bubbles with diffusion in gaseous and liquid phases.

Earlier similar problem was studied in [14]. For simplification the velocity of translatory motion of liquid around the bubble is assumed to be constant.

The latest detailed review devoted to dynamics of vapor bubbles was done in [15]. A new method of describing heat transfer between a gas bubble and liquid was developed in [16]. Vapor bubble dynamics in deuterated acetone was studied numerically in [17].

\section{Conflicts of Interest}

The author declares no conflicts of interest regarding the publication of this paper.

\section{References}

[1] Nigmatulin, R.I. (1990) Dynamics of Multiphase Media. Hemisphere, Washington DC.

[2] Nigmatulin, R.I., Khabeev, N.S. and Nagiev, F.B. (1981) Dynamics, Heat and Mass Transfer of Vapour-Gas Bubbles in a Liquid. International Journal of Heat and Mass Transfer, 24, 1033-1044. https://doi.org/10.1016/0017-9310(81)90134-4

[3] Gubaidullin, A.A., Ivandaev, A.I., Nigmatulin, R.I. and Khabeev, N.S. (1982) Waves in a Liquid with Bubbles. In: Progr. in Sci. and Techn., VINITI, Fluid Mech.

[4] Voinov, O.V. and Petrov, A.G. (1976) The Motion of Bubbles in a Liquid. In: Progr. in Sci. and Techn., VINITI, Fluid Mech.

[5] Soo, S.-L. (1967) Fluid Dynamics of Multiphase Systems. Blaisdell, Waltham, MA.

[6] Brounchtein, B.I. and Fishbein, G.A. (1977) Hydrodynamics and Heat and Mass Transfer in Disperse Systems. Khimiya, Leningrad, Saint Petersburg, Russia.

[7] Gupalo, I.P., Polyanin, A.D. and Riazantsev, I.S. (1985) Mass and Heat Transfer between Reacting Particles and Flow. Nauka, Moscow.

[8] Wittke, D.D. and Chao, B.T. (1967) Collapse of Vapor Bubbles with Translatory Motion. Journal of Heat Transfer, 89, 17-24. https://doi.org/10.1115/1.3614315

[9] Florschuetz, L.W. and Chao, B.T. (1965) On the Mechanics of Vapor Bubble Collapse. Journal of Heat Transfer, 87, 209-220. https://doi.org/10.1115/1.3689075

[10] Levich, V.G. (1962) Physicochemikal Hydrodynamics. Prentice Hall, Upper Saddle River, NJ.

[11] Moore, D.W. (1959) The Rise of a Gas Bubble in a Viscous Liquid. Journal of Fluid Mechanics, 6, 113-130. https://doi.org/10.1017/S0022112059000520

[12] Batchelor, G.K. (1967) Introduction to Fluid Dynamics. Cambridge University Press, Cambridge, England.

[13] Peaceman, D.W. and Rachford, H.H. (1955) The Numerical Solution of Parabolic and Elliptic Differential Equations. Journal of the Society for Industrial and Applied Mathematics, 3, 28-41. https://doi.org/10.1137/0103003

[14] Sagitov, R.B. and Khabeev, N.S. Fluid Dynamics, N6, 85.

[15] Prosperetti, A. (2017) Vapor Bubbles. Annual Review of Fluid Mechanics, 49, 221-248. https://doi.org/10.1146/annurev-fluid-010816-060221 
[16] Khabeev, N.S. (2017) A Method of Describing Heat Transfer Between a Gas Bubble and a Liquid. Journal of Engineering Physics and Thermophysics, 90, 353-356. https://doi.org/10.1007/s10891-017-1574-1

[17] Khabeev, R.N. and Khabeev, N.S. (2018) Vapor Bubble Dynamics in Deuterated Acetone. Journal of Engineering Physics and Thermophysics, 91,195-200. https://doi.org/10.1007/s10891-018-1735-x

\section{Nomenclature}

$\mathrm{R}$-bubble radius, $\mathrm{m}$

r-radius coordinate, $\mathrm{m}$

$\tau$-nondimensional time 